\title{
Comparison of Functional Outcomes in the Management of Intercondylar Fractures by Olecranon Osteotomy Approach versus Triceps on and Triceps off Approach
}

\author{
Made Bramantya Karna ${ }^{1}$, AA Gde Yuda Asmara ${ }^{1}$, Putu Feryawan Meregawa ${ }^{1}$, \\ Nyoman Gede Bimantara', Indra Rukmana Tri Pratistha', \\ Gede Mahardika Putra ${ }^{2}$
}

\author{
${ }^{1}$ Orthopaedics and Traumatology Department Consultant, ${ }^{2}$ Orthopaedics and Traumatology Department \\ Resident, Faculty of Medicine Udayana University and Sanglah General Hospital
}

Corresponding Author: Nyoman Gede Bimantara

\begin{abstract}
Intercondylar fracture of the distal humerus is a relatively rare and challenging intra-articular elbow fracture, with available choice of surgical treatment includes open reduction and extensive internal fixation. This systematic review aims to evaluate the effectiveness of the surgical approach regarding the functional outcome following intercondylar fracture treatment. A systematic search on literature was performed online from the inception dates to November 2020. Main outcome of interest included functional outcomes, Mayo Elbow Performance Score (MEPS) and The Disabilities of the Arm, Shoulder and Hand (DASH) Score. Animal studies, case reports, review articles and nonEnglish papers were excluded. Four articles were included in this review. The functional outcome was different among the olecranon osteotomy approach, triceps on with the triceps sparing approach and triceps off with the triceps lifting approach or the TRAP approach. Triceps on approach with triceps sparing had lower MEPS in patients above 60 years of age but satisfactory in younger patients, while olecranon osteotomy approach showed excellent MEPS rate in all age groups. Olecranon osteotomy approach gives better exposure for more accurate anatomical reduction of the intra articular fracture resulting in better functional outcome. Mean DASH score was significantly higher in Triceps Reflecting anconeus (TRAP) group. Olecranon osteotomy approach was
\end{abstract}

found to be better than triceps on approach, with better functional outcome. However, other triceps off (TRAP) did not show any significant differences in terms of functional outcome in the management of intercondylar fractures.

Keywords: intercondylar fractures, olecranon osteotomy, triceps on, triceps off, functional outcome

\section{INTRODUCTION}

Intercondylar fracture of the distal humerus is an intra-articular elbow fracture that involves soft tissue injury. This type of fracture is relatively rare $(<2 \%)$ and difficult to treat because of the location of the epiphyses. Complete fractures result from impaction of the ulna proximal to the articular portion (trochlea, capitellum) of the distal humerus, and may occur with the elbow flexed or extended. Due to the fact that these fractures are rare, special treatment schemes are difficult to come up with. ${ }^{1}$

Treatment consists of assessing the mechanism of the injury, determining diagnostic modalities, and developing a clinical approach that will allow full restoration of elbow mobility. Each approach should aim to mobilize the elbow joint to avoid heterotopic hardening and hardening. Immobilization can only be 
performed in situations where the fracture has not shifted or as a temporary treatment in certain circumstances. Normal function can be difficult to restore if the joint is deformed due to a malunion and/or is stiffened by contracture of the capsular and ligaments or heterotopic ossification. ${ }^{1}$

The choice of management in patients with intercondylar fractures can be performed operatively using open reduction and extensive internal fixation. Nonoperative treatment of intra-articular fractures of the distal humerus usually results in loss of elbow motion and permanent disability. ${ }^{2}$ Olecranon osteotomy is considered the "gold standard" treatment for distal humerus fractures. Fixation for complex fractures can consist of reconstruction plates or locking compression plates, with one plate being placed on each column to neutralize disassembly forces, especially rotational ones. Complications following surgery include loss of reduction, implant failure, non-union, malunion, ulnar nerve neuropathy, elbow stiffness, and heterotopic ossification. ${ }^{3}$

The most effective approach to treating patients with intercondylar fractures is unclear and may depend on several factors, such as the complexity of the fracture and patient characteristics. In a systematic review aimed to evaluate the effectiveness of the surgical approach by looking at the functional outcome of the elbow in the treatment of intercondylar fractures of the distal humerus.

\section{MATERIAL AND METHODS Search Strategy}

Focused literature searches were primarily conducted using the Google Scholar and Pubmed/MEDLINE, from their inception dates to November 2020. To optimize sensitivity and specificity of the search method and identify all research, using the keywords listed below in combination with Boolean "AND" and "OR" phrases. Search terms: "humerus intercondylar fracture", "distal humerus fracture" "surgical approach", "triceps on", "triceps off", "olecranon osteotomy", "functional outcome".

\section{Inclusion Criteria}

One reviewer screened the search results. Operative management of patients with intercondylar fracture, olecranon osteotomy, triceps on, triceps off and functional outcome in this systematic review. Patients without distal humerus fracture, operative management using the lateral approach, the medial approach and the medial approach were excluded. Other than studies using English were excluded in this systematic review (Table 1).

Table 1: PICO Table

\begin{tabular}{|c|c|c|}
\hline $\begin{array}{c}\text { Study } \\
\text { Component }\end{array}$ & Inclusion & Exclusion \\
\hline Population & $\begin{array}{ll}\text { - } & \text { Patients with intercondylar fracture } \\
\text { - } & \text { Patient with distal humerus fracture }\end{array}$ & $\begin{array}{ll}- & \text { Animal studies } \\
\text { - } & \text { Patient without distal humerus fracture } \\
\end{array}$ \\
\hline $\begin{array}{l}\text { Intervention and } \\
\text { Comparison }\end{array}$ & $\begin{array}{ll}- & \text { Olecranon osteotomy } \\
\text { - } & \text { Triceps On } \\
\text { - } & \text { Triceps Off } \\
\text { - } & \text { Posterior Approach } \\
\end{array}$ & $\begin{array}{ll}\text { - } & \text { Other methods of treatment } \\
\text { - } & \text { Lateral approach } \\
\text { - } & \text { Medial approach } \\
\text { - } & \text { Anterior approach } \\
\end{array}$ \\
\hline Outcome & $\begin{array}{ll}\text { - } & \text { Functional outcome } \\
\text { - } & \text { Mayo Elbow Performance Score (MEPS) } \\
& \text { The Disabilities of the Arm Shoulder and Hand (DASH) Score }\end{array}$ & \\
\hline Publication & $\begin{array}{ll}\text { - } & \text { Studies published in English } \\
\text { - } & \text { Listed in Pubmed or Google Scholar Archives }\end{array}$ & $\begin{array}{ll}\text { - } & \text { Proceedings, presentations } \\
\text { - } & \text { Abstracts, editorials, letters } \\
\text { - } & \text { Duplicate publications of the same study } \\
\text { - } & \text { Studies published in language other than English } \\
\end{array}$ \\
\hline Study Design & Retrospective study - Randomized control trial & Case reports, review articles \\
\hline
\end{tabular}

\section{Quality Evaluation}

This was done, based on inclusion criteria the authors filtered eligible studies through titles and abstracts. Then, the authors screened the complete studies from all the studies that were collected. The author is looking for publications that are very relevant to be included in this research. 
The author also assessed the quality of the study.

\section{RESULTS}

The electronic search returned 25 records, after removing duplicate results. On the basis of screening of abstracts and titles, a total of 20 records were excluded. The remaining articles were studied by the authors independently based on the extracted full text. This selection process resulted in the final four articles for inclusion in the systematic review and is depicted in Figure 1. The remaining 4 studies were eventually included, consisting of 2 retrospective study, 1 comparative study and 1 randomised controlled trial. The details of this study are listed in Table 2.
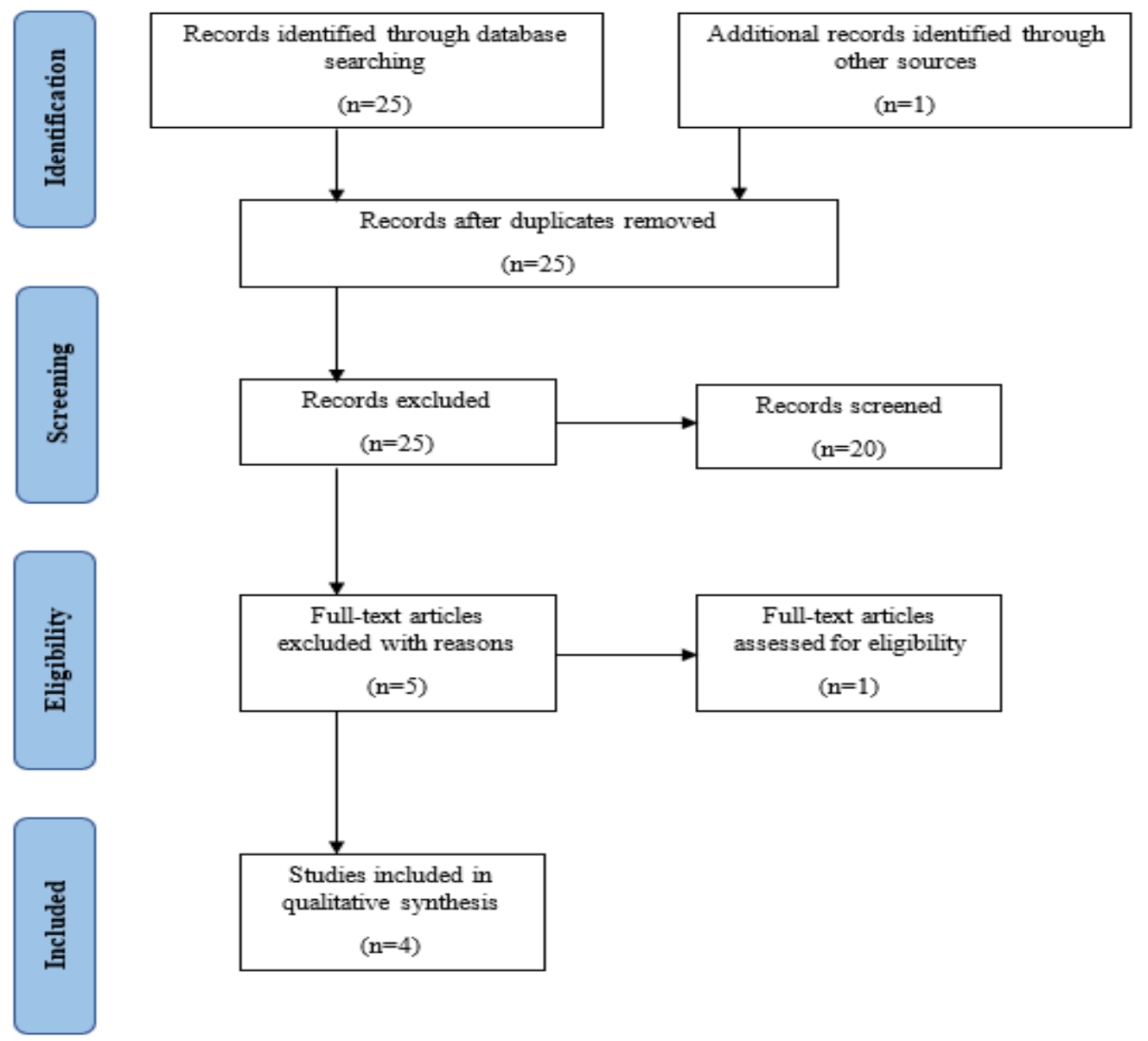

Figure 1 PRISMA flow-chart

Table 2: Characteristic of the studies

\begin{tabular}{|c|l|l|l|c|}
\hline No & \multicolumn{1}{|c|}{ Reference } & \multicolumn{1}{|c|}{ Journal } & \multicolumn{1}{|c|}{ Study Design } & $\begin{array}{c}\text { Level of } \\
\text { Evidence }\end{array}$ \\
\hline 1 & Khalid et al., 2015 & Journal of Pakistan Medical Association & Randomised controlled study & III \\
\hline 2 & Azboy et al., 2015 & Turkish Journal of Trauma \& Emergency Surgery & Retrospective cohort study & III \\
\hline 3 & Elmadag et al., 2014 & European Journal of Orthopaedic Surgery and Traumatology & Retrospective cohort study & III \\
\hline 4 & Chen et al., 2011 & Annals of Medicine & Comparative study & III \\
\hline
\end{tabular}

According to a study by Chen et.al, it was found that the triceps on approach with triceps sparing had lower functional results where they showed more loss of extension (mean 22.98, range 0-558) in the arm and the Mayo Elbow Performance Score (MEPS) less than $40 \%$ at the age above 60 years. Whereas in patients aged 41 and 59 years, the MEPS rate increased to $80 \%$ and $100 \%$ at the age under 40 years. Fisher's exact test showed that in the triceps sparing group, the excellent MEPS rate in patients over 60 years of age was significantly lower than in patients less than 40 years of age $(P=0.0207)$. In contrast, the excellent MEPS rate remains above $80 \%$ in 
all age groups of patients treated with the olecranon osteotomy approach. ${ }^{4}$

In a study conducted by Khalid et.al found that the Olecranon osteotomy approach gives better exposure for more accurate anatomical reduction of the intra articular fracture that results in better functional outcome. In the management using olecranon osteotomy, the range of motion (ROM) of the patients was $70.7 \%$ of the patients had arc 50-100 degree. In the management using triceps sparing, $58.7 \%$ of patients had arc 50-100 degree. In the management using olecranon osteotomy $89.3 \%$ of patients had a satisfactory functional outcome and only $10.7 \%$ of patients had an unsatisfactory outcome, whereas in the management using triceps sparing, $70.7 \%$ of patients had a satisfactory functional outcome and $29.3 \%$. $\%$ of patients have an unsatisfactory outcome. ${ }^{2}$

Meanwhile, another study conducted by Elmadag et.al compared the functional outcome between the triceps off approach, namely triceps lifting and olecranon osteotomy. MEPS obtained in the management using olecranon osteotomy was excellent in 10 , good in 8 , moderate in 3 and poor in 1 patient, with a mean MEPS of $85 \pm 13.2$. The results for treatment using triceps lifting were excellent in 6 , good in 14 , moderate in 7 and poor in 5 patients, with a mean score of $76.7 \pm 13.4$. The mean MEPS was a significant different between the groups with $\mathrm{p}<0.05$. Where the olecranon osteotomy approach has better functional results than using the triceps lifting approach in patients with distal humerus fracture. In this study also, the range of joints was evaluated at the end of the postoperative follow-up visit, which averaged $99.3 \pm 11.6$ elbow flexion in the management of the patients using the olecranon osteotomy approach and the limitation of elbow extension was 13.4 \pm 9.3 . These values were $88.8 \pm 12.3$ and 22.3 \pm 11.1 respectively in the group using the triceps lifting approach, and there was a significant difference $(\mathrm{p}<0.005)^{5}$
In a study conducted by Azboy et.al which compared the functional outcome between the olecranon osteotomy approach with other triceps off approaches, namely Triceps Reflecting anconeus (TRAP) in terms of MEPS values, the mean MEPS was 85.9 (range 55-100) in the TRAP group, whereas in the olecranon osteotomy group was 83.5 (range 55-100). Although the mean MEPS was higher in the TRAP group, there was no significant difference between the two groups $(p=0.412)$. Judging from the mean score of The Disabilities of the Arm Shoulder and Hand (DASH) was 15.6 (range, 0-48) in the TRAP group, while in the olecranon osteotomy group it was 20.1 (range 4-57). Although the mean DASH score was higher in the TRAP group, no significant differences were detected between the two groups $(p=0.201)$. Judging from the mean range of motion (ROM) of the elbow was $108^{\circ}$ (range $70^{\circ}-140^{\circ}$ ) in the TRAP group, while the olecranon osteotomy group was $98^{\circ}$ (range $70^{\circ}-115^{\circ}$ ). There was a significant difference observed between the two groups in terms of the overall mean elbow motion $(\mathrm{p}=0.038){ }^{6}$

\section{DISCUSSION}

Intercondylar fracture is a relatively rare fracture, and the management of intercondylar fracture is unclear. The management goal of this fracture is to restore function to the elbow joint of the patient with an intercondylar fracture. This systematic review evaluates the effectiveness of different surgical approaches on the functional outcome of the elbow in the treatment of fractures of the distal humerus. ${ }^{1}$

This systematic review evaluated the effectiveness of different surgical approaches on the functional outcome in the management of intercondylar humerus fractures. The systematic review included 4 studies, 2 retrospectives, 1 comparative study and 1 randomized control trial.

In this discussion, we found that the management of intercondylar fractures using the olecranon osteotomy approach had 
a better functional outcome than the triceps on approach or the triceps-sparing approach. This is as reported by Gang Chen et.al where they found that the triceps on approach with triceps sparing had a lower functional outcome in that they showed more loss of extension (mean 22.98, range $0-558)$ in the arm and a MEPS rate of less than $40 \%$ at the above age. 60 years. Whereas the MEPS rate with the olecranon osteotomy approach was $>80 \%$ for all ages ${ }^{4}$. The same thing was reported by Khalid et.al. where the olecranon osteotomy approach provides a better functional outcome than using the triceps sparing approach. The use of the triceps sparing approach can cause fibrosis and limited range of motion of the elbow joint due to the separation or reflection of the triceps, so the functional results are less satisfactory. ${ }^{2}$

In a study conducted by Elmadag et.al, it was found that the triceps lifting or triceps off approach obtained a lower functional outcome compared to the olecranon osteotomy approach seen from the MEPS score and better extension and flexion elbow motion arc ${ }^{5}$. Different results obtained with other triceps off approaches, namely TRAP in a study conducted by Azboy et.al found that the TRAP approach was more successful than olecranon osteotomy in terms of overall mean elbow motion $(\mathrm{p}=0.038)$. When DASH score and MEPS score were examined, it was observed that the results were better for the TRAP group, but in this study there was no statistically significant difference between olecranon osteotomy and TRAP $(\mathrm{p}=0.234)$ $(\mathrm{p}=0.403)$, respectively. ${ }^{6}$

In this study, it was found that the functional outcome was different using the olecranon osteotomy approach, triceps on with the triceps sparing approach and triceps off with the triceps lifting approach or the TRAP approach. Further study should be done to see the functional outcome in patients with intercondylar fractures with the olecranon osteotomy approach, triceps on and triceps off.

\section{CONCLUSION}

In this systematic review, it was found that the management of patients with intercondylar fractures using the olecranon osteotomy approach was better than using the triceps on approach, namely triceps sparing seen from the functional outcome. The olecranon osteotomy approach also has a better functional outcome than using the triceps off approach such as triceps lifting. However, other triceps off approaches such as TRAP did not show any significant differences in terms of the functional outcome in the management of patients with intercondylar fractures.

\section{Acknowledgement: None}

Conflict of Interest: None declared

\section{Source of Funding: None}

\section{Ethical Approval: Not required}

\section{REFERENCES}

1. Chen $\mathrm{H}$, Li D, Zhang J, Xiong $X$. Comparison of treatments in patients with distal humerus intercondylar fracture: a systematic review and meta-analysis. Annals of Medicine. 2017 Oct 3;49(7).

2. Usman Khalid M, Muhammad Saeed K, Akhter M. Introduction A comparison of functional outcome of intercondylar fracture of distal humerus managed by olecranon osteotomy approach versus triceps sparing approach in adults. Journal of Pakistan Medical Association. 2015;65(11):119.

3. Lakhey S, Sharma S, Pradhan RL, Pandey BK, Manandhar RR, Rijal KP. Osteosynthesis of intercondylar humerus fracture using Bryan and Morrey approach. Journal of the Nepal Medical Association. 2010;

4. Chen G, Liao Q, Luo W, Li K, Zhao Y, Zhong D. Triceps-sparing versus olecranon osteotomy for ORIF: Analysis of 67 cases of intercondylar fractures of the distal humerus. Injury. 2011 Apr;42(4).

5. Elmadag M, Erdil M, Bilsel K, Acar MA, Tuncer N, Tuncay I. The olecranon osteotomy provides better outcome than the triceps-lifting approach for the treatment of distal humerus fractures. European Journal 
Made Bramantya Karna et.al. Comparison of functional outcomes in the management of intercondylar fractures by olecranon osteotomy approach versus triceps on and triceps off approach

of Orthopaedic Surgery \& Traumatology. 2014 Jan 21;24(1).

6. Azboy I. The comparison of tricepsreflecting anconeus pedicle and olecranon osteotomy approaches in the treatment of intercondylar fractures of the humerus. Turkish Journal of Trauma and Emergency Surgery. 2015;
How to cite this article: Karna MB, AA Gde Yuda Asmara, Meregawa PF. Comparison of functional outcomes in the management of intercondylar fractures by olecranon osteotomy approach versus triceps on and triceps off approach. International Journal of Science \& Healthcare Research. 2021; 6(4): 36-41. DOI: https://doi.org/10.52403/ijshr.20211006 